\title{
Awake Nasal Fiberoptic Intubation in Diffuse Para-pharyngeal Abscess
}

Case

Abdul Jabbar bin Ismail ${ }^{1,2}$, Wan Aireene Wan Ahmeed ${ }^{3}$, Rhendra Hardy Mohd

Article

Zaini $^{1,2}$, Wan Fadzlina Wan Muhd Shukeri ${ }^{1,2}$, Mohd Zulfakar Mazlan ${ }^{1,2}$

${ }^{I}$ Department of Anaesthesiology and Intensive Care, School of Medical Sciences, Universiti

Sains Malaysia, 16150 Kota Bharu, Kelantan, Malaysia, ${ }^{2}$ Department of Anaesthesiology

and Intensive Care, Hospital Universiti Sains Malaysia, 16150 Kubang Kerian, Kelantan,

Malaysia, ${ }^{3}$ Department of Radiology, Hospital Universiti Sains Malaysia, 16150 Kubang

Kerian, Kelantan, Malaysia

\begin{abstract}
This is a case report on awake fibreoptic intubation for a patient with a deep neck space infection. Intubation in this group of patients is considered difficult. It is challenging to anaesthesiologists, emergency physicians and otorhinolaryngologists because there is no consensus for airway management in these patients. We present a 30 -year-old gentleman with swelling over the right cheek, difficulty breathing and severe trismus. He had a history of toothache one month prior to admission. Upon clinical examination, there was a diffuse swelling over the right mandible. Other examinations were unremarkable. Provisional diagnosis of a right para-pharyngeal abscess was made secondary to a possible infected right lower $3^{\text {rd }}$ molar, with a differential diagnosis of a right parotid abscess with para-pharyngeal extension. Radiological assessment using computed tomography (CT) of the head and neck region showed an abscess over the right para-pharyngeal area, soft palate and right submandibular region. The narrowest part of the airway was at the region posterior to the soft palate, measuring approximately $1 \mathrm{~cm}$. All staff and equipment were prepared for intubating a difficult airway. The patient was transferred to the control environment (operation theatre) for intubation. Awake nasal fibreoptic intubation (AFOI) was successfully performed for this patient using intravenous dexmedetomidine alone as the sedative.
\end{abstract}

Key Words: Awake nasal fibreoptic intubation, dexmedetomidine, para-pharyngeal abscess, sedation.

Received: 13 April 2020, Accepted: 11 September 2020

Corresponding Author: Mohd Zulfakar Mazlan, MD, Department of Anesthesiology and Intensive Care, School of Medical Sciences, Universiti Sains Malaysia Health Campus, 16150, Kota Bharu, Kelantan, Tel.: +609-7676104 / 012-9091447, E-mail: zulfakar@usm.my

ISSN: 2090-0740, March 2021 Vol.22, No.1

\section{INTRODUCTION}

Deep neck space infections refer to infections involving the extensive network of spaces in the neck region. In view of the distorted anatomy of the airway due to abscess and oedema formation as well as difficulty in opening the mouth and neck movement, awake fibre optic intubation (AFOI) with tracheostomy standby is a preferred mode of inducing anaesthesia ${ }^{[1,2]}$. Although tracheostomy under local anaesthesia was previously the gold standard in difficult airway management ${ }^{[3]}$, awake fibreoptic intubation with either oral or nasal intubation has been gaining acceptance by anaesthesiologists ${ }^{[4,5]}$. Proper preparation is the key to successful airway management, including anaesthesiologists trained in awake fibreoptic intubation and the required perioperative staff and equipment to manage the difficult airway.

Although deep neck space infection is a clinical diagnosis, computed tomography (CT) is commonly used in the assessment of the extent and localization of these infections for precise intraoperative surgical drainage ${ }^{[6,7]}$. The CT scan can also be used to determine and assess patency along the whole tract of the airway. Measurement of the airway diameter at various levels can also be performed prior to airway management, which may guide endotracheal tube size selection. Thus, if feasible, CT scans can play a vital role in increasing the probability of success in managing life-threatening airways in patients with deep neck space infections.

\section{CASE REPORT:}

A 30-year-old gentleman presented with a chief complaint of pain and swelling on the right cheek, difficulty breathing and persistent high-grade fever for the past 5 days. The symptoms started with a mild toothache of the right lower $3^{\text {rd }}$ molar for 1 month and were associated 
with intermittent fever. The patient claimed to have taken 2 courses of oral antibiotics but was unable to name them. However, the pain did not resolve and worsened over the past 5 days, but this time was associated with swelling over the cheek, below the ear, and at the angle of the right jaw and pain over the right neck. He was unable to open his mouth fully or to tolerate solid food orally, had odynophagia and dysphagia, and had been drinking only water for the past 2 days.

Upon clinical examination, the patient was fully conscious, alert and oriented to time, place and person, looked lethargic, pink, and mildly tachypnoeic with a respiratory rate of 24 breaths per minute with pulse oximetry of $96 \%$ on room air. The pulse volume was good, the blood pressure was $130 / 94 \mathrm{~mm} \mathrm{Hg}$, the heart rate was 119 per minute, and the temperature was $38.8^{\circ} \mathrm{C}$. He had a swollen right preauricular region, extending superiorly to the right ear lobe, anteriorly to the anterior border of the masseter, inferiorly to the level II and III cervical lymph node regions and posteriorly to the posterior border of the parotid gland. (Figure 1)

He was also noted to have severe trismus, only able to open his mouth by approximately $2 \mathrm{~cm}$ (approximately the size of 1 finger breadth). Flexible endoscopy performed in the ENT clinic revealed a crowded oropharynx with a swollen mucosal wall and medialization of the right lateral pharyngeal wall obliterating the right pyriform fossa. The bilateral arytenoid cartilages were also swollen, but the epiglottis and the vocal cord were normal.

Blood examinations during the patient presentation were all within normal, including leucocyte count, except for pus culture from the operation theatre, which revealed Streptococcus viridians. A plain radiograph of the neck on a lateral view was performed and showed homogenous soft tissue opacities overlying the nasopharynx region at the level of the $\mathrm{C} 1$ and $\mathrm{C} 2$ vertebrae with loss of cervical lordosis. (Figure 2) In view of the severe nature of his swollen and oedematous region of the pharyngeal area, contrast-enhanced CT of the neck region was performed. The CT scan showed a right submandibular and sublingual heterogeneously enhancing abscess measuring $3.3 \mathrm{~cm}$ antero-posteriorly, $1.2 \mathrm{~cm}$ width, and $3.0 \mathrm{~cm}$ craniocaudally extending superior and medially into the right parapharyngeal and masticator space. (Figure 3) The oropharynx was also compressed and displaced to the contralateral side. However, the patency of the airway was still preserved with the narrowest of $1 \mathrm{~cm}$ just posterior to the soft palate.

The patient consented and was prepared for the awake nasal fibreoptic intubation procedure. He was given nebulization with $4 \mathrm{ml}$ of $2 \%$ lidocaine over 20 minutes, followed by nasal insufflation with cotton soaked with a $4 \%$ cocaine solution over 10 minutes at the more patent nostril. Blood pressure was stable and was measured every 5 minutes throughout the procedure. The saturation of the partial pressure of oxygen $(\mathrm{SpO} 2)$ was continuously monitored. An intravenous dexmedetomidine infusion was started to aid in maintaining sedation while keeping the patient spontaneously breathing. A loading dose of 1 $\mathrm{mcg} / \mathrm{kg}$ over 10 minutes was started and maintained at $0.5 \mathrm{mcg} / \mathrm{kg} / \mathrm{hr}$. A face mask with a special hole for the flexible fibreoptic scope to pass through was applied onto the patient's face to provide continuous $100 \%$ oxygen at a flow rate of $15 \mathrm{~L} / \mathrm{min}$. The flexible fibreoptic scope was introduced through the left anterior nasal aperture and guided under direct vision along the floor of the nose. As expected, difficulty was encountered at the level posterior to the soft palate as a result of airway narrowing, and several manoeuvres needed to be performed to pass through the small opening to reach the oropharynx.

Upon viewing the larynx, (Figure 5) 2\% lidocaine was injected over the arytenoid, epiglottis $(4 \mathrm{ml})$, and subsequently the vocal cord $(2 \mathrm{ml})$ and the inner mucosa of trachea $(2 \mathrm{ml})$. During the "spray as you go" technique of local anaesthetic application, the patient required up to $8 \mathrm{ml}$ of $2 \%$ lidocaine, which was double the dose normally required, to effectively suppress the reflexes of the larynx and vocal cord. Difficulty was encountered when the reinforced endotracheal size of $7.5 \mathrm{~mm}$ internal diameter was unable to pass through the narrowed nasopharynx. A new size of $7.0 \mathrm{~mm}$ was used, and intubation was finally successful.

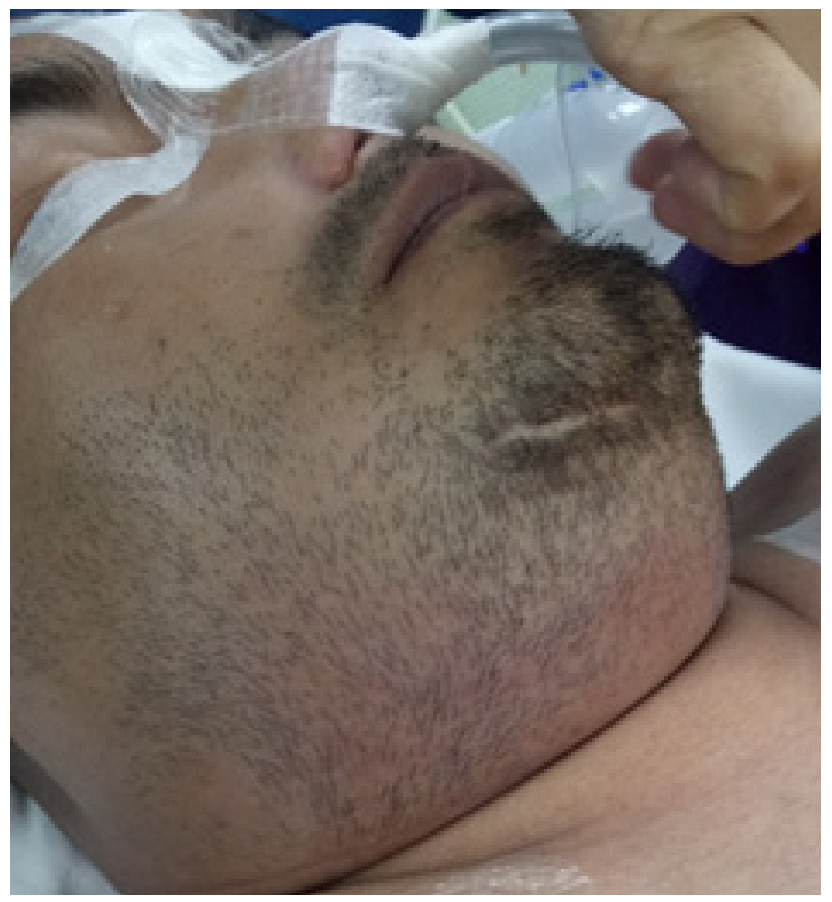

Fig. 1: Right submandibular swelling and redness 


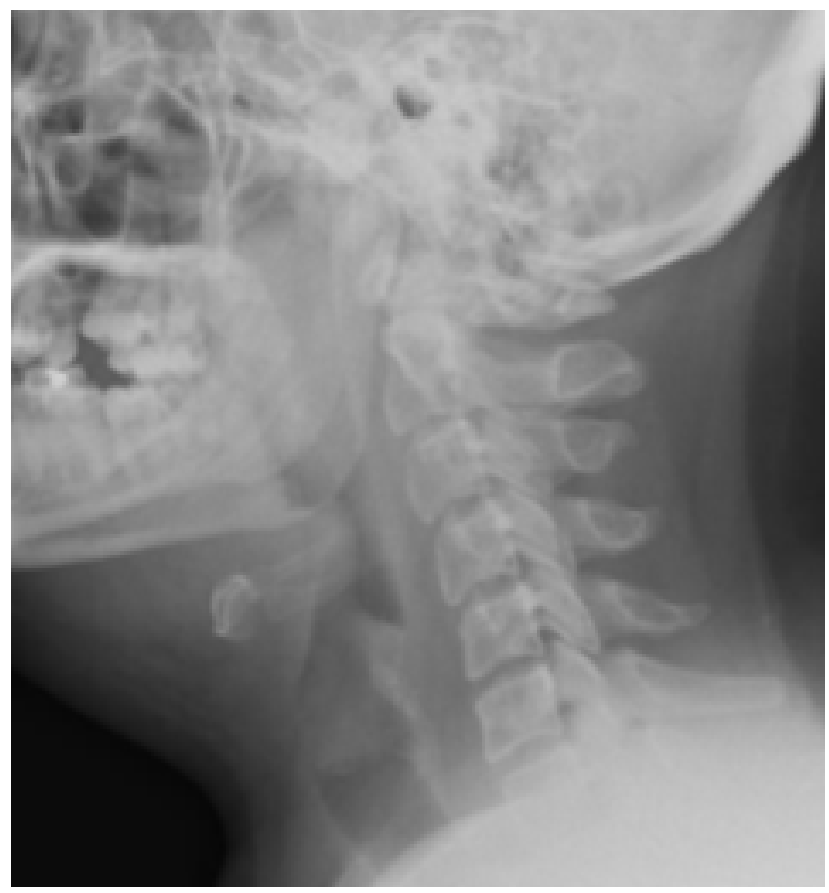

Fig. 2: Plain radiograph of the neck (lateral view) showing soft tissue opacities overlying the nasopharynx and oropharynx region at the level of the $\mathrm{C} 1$ and $\mathrm{C} 2$ vertebrae.

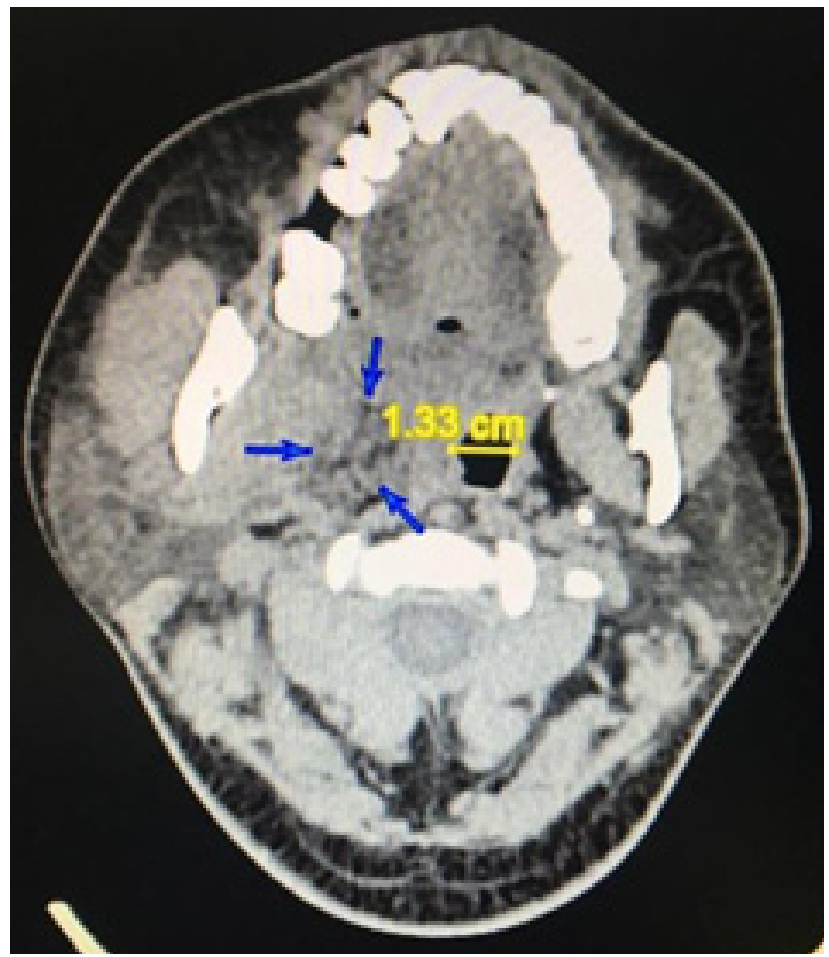

Fig. 3: Hypodense area at the right submandibular and sublingual region measuring $3 \mathrm{~cm}(\mathrm{AP}) \times 1 \mathrm{~cm}(\mathrm{~W}) \times 3 \mathrm{~cm}(\mathrm{CC})$ with the oropharynx compressed to the contralateral side by a distance of $1.3 \mathrm{~cm}$.

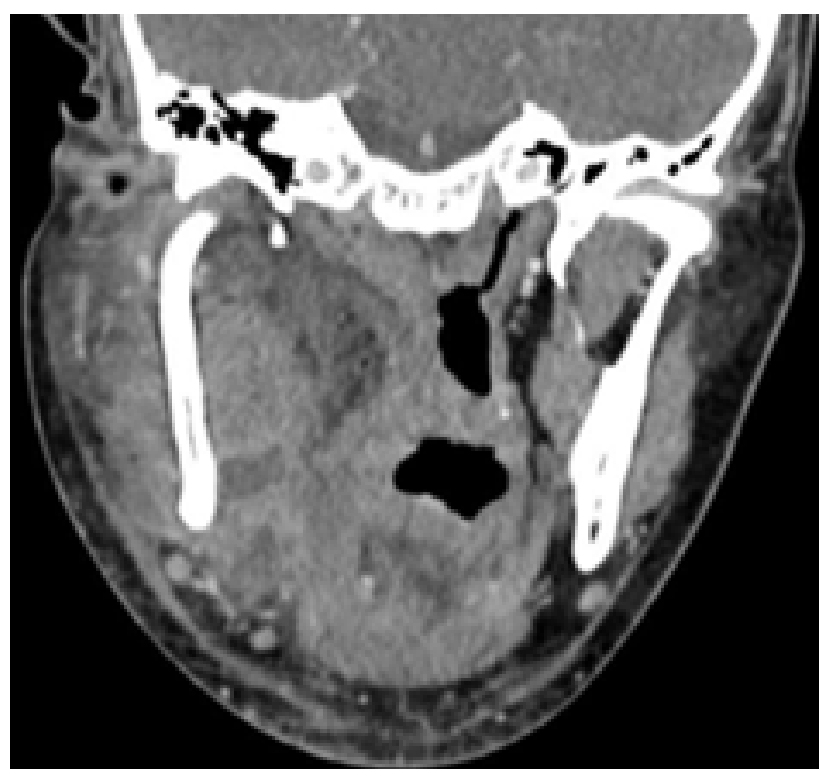

Fig. 4a

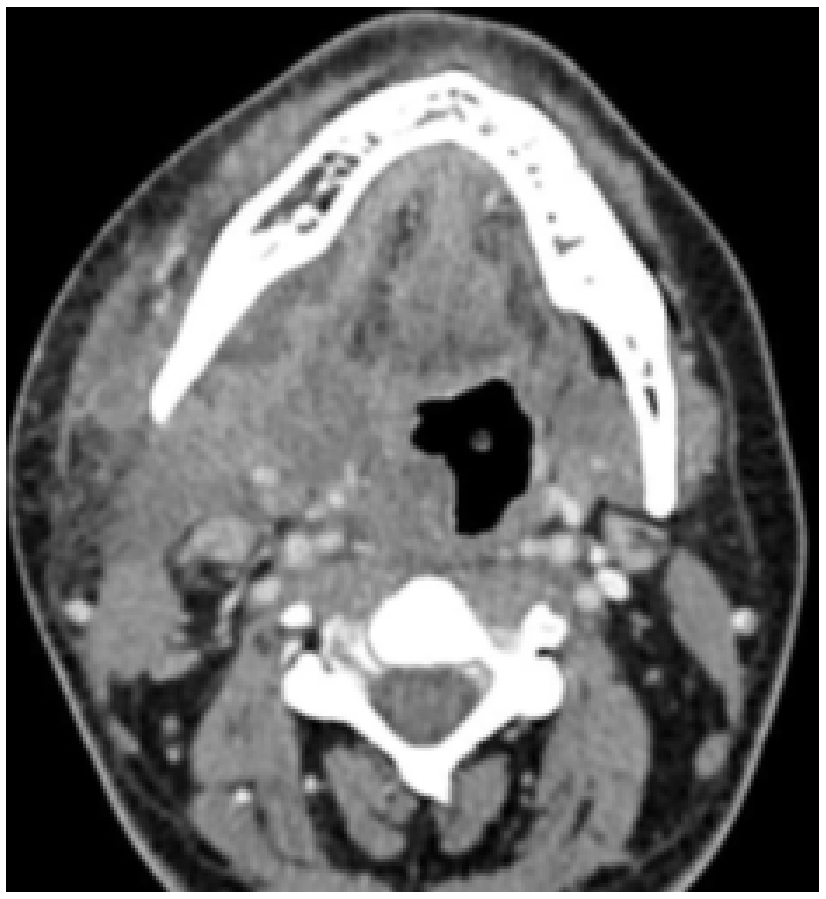

Fig. 4b: Contrast enhanced neck CT Coronal and axial views (A and B) of a contrast enhanced neck $\mathrm{CT}$ show a right submandibular and sublingual heterogeneously enhancing abscess (thin arrow) extending superior and medially into the right parapharyngeal and masticator space (thick arrow). The oropharynx was compressed and displaced to the contralateral side. 


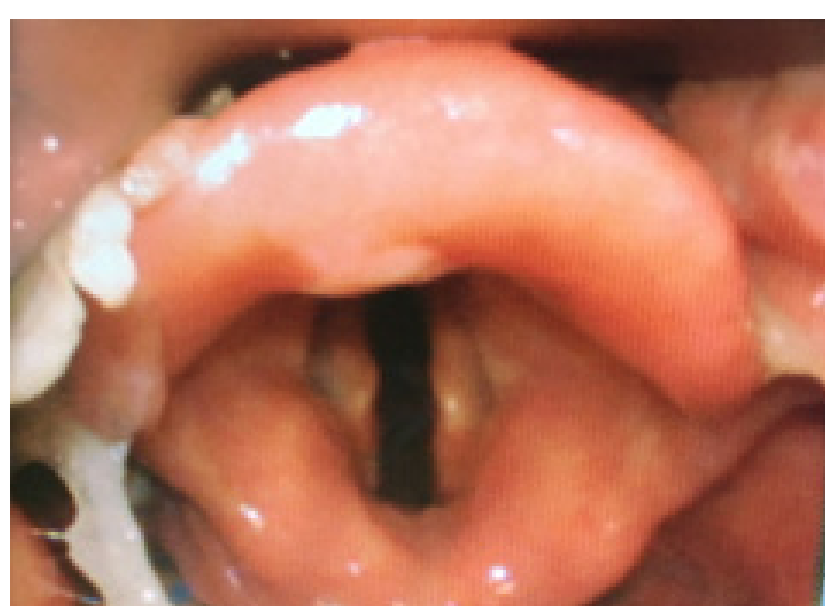

Fig. 5: Epiglottis, vocal cords and aryepiglottic folds

Endotracheal placement and the depth of intubation were confirmed with repeat flexible fibreoptic imaging. A loading dose of intravenous propofol was given to induce general anaesthesia, and a muscle paralytic was given to facilitate the surgery immediately whenever the ETT was confirmed to be inside the trachea during the repeat flexible fibreoptic examination. Intravenous dexamethasone $(8$ $\mathrm{mg}$ ) was given to reduce the generalized swelling of the airway, and adequate intravenous morphine was given as an analgesic. Postoperatively, the patient was intubated for 48 hours. The intravenous antibiotics cefuroxime and metronidazole were continued due to the culture from the pus aspirated from the abscesses intraoperatively indicating a growth of Streptococcus viridians.

\section{DISCUSSION}

Deep neck space infections, among which include para-pharyngeal abscesses and Ludwig's angina (submandibular abscess), have been associated with airway compromise and difficulty in managing the airway using awake fibre optic intubation ${ }^{[4]}$, and in some cases, even tracheostomy under local anaesthesia can be difficult ${ }^{[8]}$. In our patient, both a para-pharyngeal abscess and a submandibular abscess were present at the same time, as well as soft palate swelling, which further complicated airway management.

Sudden airway compression during awake fibreoptic intubation had been documented in a previous case report, when the patient had an unexpected swollen para-pharyngeal mucosa, in addition to goitre, which further compressed the airway, subsequently needing a percutaneous tracheostomy ${ }^{[9]}$. Another case report depicted the unanticipated loss of airway reflexes, which led to total airway obstruction after airway topicalization was given ${ }^{[10]}$. Unanticipated incidents with no appropriate interventions could ultimately endanger patients' lives and lead to hypoxic events.
Thus, proper preparation is required to prevent such an occurrence from happening.

Pre-operative planning, including the assessment of airway patency and the measurement of airway diameter, can be of paramount importance, especially when CT scans are available and can be feasibly performed prior to surgery ${ }^{[1]}$. Although preparation and management are necessary to reduce the possibility of mishaps, doctors might make mistakes, which can be life-threatening. In this case, a smaller endotracheal tube the appropriate words is should have been chosen. The outer diameter of the endotracheal tube should be smaller than the measured size of the narrowest part of the airway to ensure that the first attempt at intubation is the best attempt. Repeated airway manoeuvres with multiple intubations may lead to more swelling and oedema and may further complicate the procedure of securing the airway. Repeated attempts at intubation have been shown to be significantly associated with adverse events ${ }^{[12]}$. Failure to secure the airway in this case would have been life-threatening and catastrophic, as the airway was already compromised with severe deep neck space infections.

In the case of patients presenting with difficult airway management and deep neck space infections, it is unfortunate that no consensus is present, and even practice guidelines for difficult airway management by the American Society of Anaesthesiologists does not address this challenge or make recommendations in cases such as this. A study was conducted to observe the multiple ways in which an anaesthetist would use in managing the airway of a patient with deep neck space infection, showing that no current consensus is present ${ }^{[4]}$.

Despite the overwhelming number of case studies on the use of awake fibreoptic intubation as a safe technique in airway management for deep neck space infection, there are still no proper clinical trials recommending which techniques should be employed in this kind of case. The reason may be because of the heterogeneity of the presentations of deep neck space infection, which makes it difficult to perform a pragmatic clinical study from the point of view of airway management.

\section{CONCLUSION}

Awake fibreoptic intubation via the nasal route under monitored anaesthesia care with a dexmedetomidine loading dose of $1 \mathrm{mcg} / \mathrm{kg}$ over 10 minutes and maintenance with $0.5 \mathrm{mcg} / \mathrm{kg} / \mathrm{hr}$ is the preferred mode of anaesthesia for diffuse parapharyngeal abscesses. 


\section{CONFLICT OF INTEREST}

There are no conflicts of interest.

\section{ACKNOWLEDGEMENTS}

This case report was written based on clinical experience during anaesthesia practice, and proper informed consent, signed by the patient, was obtained for the purpose of this case report submission. We affirm the honest, accurate and transparent account of the study, case or topic being reported; no important aspects have been omitted.

The authors also have no conflicts of interest in this case report. The English editing fee for the purpose of publication of this case report is funded by Universiti Sains Malaysia Bridging Grant 304/6316494.

\section{REFERENCES}

1. Karkos PD, Leong SC, Beer H, Apostolidou MT, Panarese A. Challenging airways in deep neck space infections. American journal of otolaryngology. 2007;28(6):415-8.

2. Kataria G, Saxena A, Bhagat S, Singh B, Kaur M, Kaur G. Deep Neck Space Infections: A Study of 76 Cases. Iranian Journal of Otorhinolaryngology. 2015;27(81):293-9.

3. Kaluskar S, Bajaj P, Bane P. Deep space infections of neck. Indian Journal of Otolaryngology and Head \& Neck Surgery. 2007;59(1):45-8.

4. Cho SY, Woo JH, Kim YJ, Chun EH, Han JI, Kim $\mathrm{DY}$, et al. Airway management in patients with deep neck infections: A retrospective analysis. Medicine. 2016;95(27)

5. Ovassapian A, Tuncbilek M, Weitzel EK, Joshi CW. Airway management in adult patients with deep neck infections: a case series and review of the literature. Anesthesia and analgesia. 2005;100(2):585-9.
6. Wang B, Gao BL, Xu GP, Xiang C. Images of deep neck space infection and the clinical significance. Acta radiologica (Stockholm, Sweden : 1987). 2014;55(8):945-51.

7. Lawrence R, Bateman N. Controversies in the management of deep neck space infection in children: an evidence-based review. Clinical Otolaryngology. 2017 Feb;42(1):156-63.

8. Tubachi J HA, Pradeep DC, Nayak P. Surgical Management of Parapharyngeal Abscess. Int $\mathrm{J}$ Otorhinolaryngol Clin. 2012;4(3):122-4.

9. Shaw IC, Welchew EA, Harrison BJ, Michael S. Complete airway obstruction during awake fibreoptic intubation. Anaesthesia. 1997;52(6):582-5.

10. Ho AM, Chung DC, To EW, Karmakar MK. Total airway obstruction during local anesthesia in a nonsedated patient with a compromised airway. Canadian journal of anaesthesia $=$ Journal canadien d'anesthesie. 2004;51(8):838-41.

11. Apfelbaum JL, Hagberg CA, Caplan RA, Blitt CD, Connis RT, Nickinovich DG, et al. Practice Guidelines for Management of the Difficult AirwayAn Updated Report by the American Society of Anesthesiologists Task Force on Management of the Difficult Airway. Anesthesiology. 2013;118(2):251-70.

12. Hasegawa K, Shigemitsu K, Hagiwara Y, Chiba T, Watase H, Brown CA, 3rd, et al. Association between repeated intubation attempts and adverse events in emergency departments: an analysis of a multicenter prospective observational study. Annals of emergency medicine. 2012;60(6):749-54.e2. 